\title{
In-hospital outcomes of neonates with hypoxic-ischemic encephalopathy receiving extracorporeal membrane oxygenation
}

\author{
Prashant Agarwal $^{1} \cdot$ Deniz Altinok $^{2} \cdot$ Jagdish $^{\text {Desai }}{ }^{3} \cdot$ Christina Shanti $^{4} \cdot$ Girija Natarajan $^{1}$
}

Received: 21 November 2018 / Revised: 31 January 2019 / Accepted: 1 February 2019 / Published online: 6 March 2019

(c) Springer Nature America, Inc. 2019

\begin{abstract}
Objective To determine in-hospital outcomes of neonates with hypoxic ischemic encephalopathy (HIE) requiring extracorporeal membrane oxygenation (ECMO).

Study design Single-center retrospective study from 2005 to 2016 of neonates $\geq 35$ weeks gestation with moderate/severe HIE, requiring ECMO for persistent pulmonary hypertension of newborn (PPHN).

Results Our cohort $(n=20)$ received therapeutic hypothermia for moderate $(n=12)$, severe $(n=5)$, or undocumented severity $(n=3)$ of HIE. During ECMO, 30\% $(n=6)$ infants developed intracranial hemorrhage at a median (IQR) duration of 24 (20) hours. Sixteen (80\%) infants survived to discharge, among which 15 had MRI performed; 47\% $(n=7)$ had normal MRI, $20 \%(n=3)$ had intracranial hemorrhage and 13\% $(n=2), 13 \%(n=2)$ and $7 \%(n=1)$ had NICHD stage 1,2 , and 3 pattern of brain injury respectively.

Conclusions In this high-risk population of neonates, use of ECMO was safe and efficacious as demonstrated by survival and outcomes.
\end{abstract}

\section{Introduction}

Neonatal hypoxic ischemic encephalopathy (HIE) is associated with significant mortality and adverse neurodevelopmental outcomes. Therapeutic hypothermia has been shown to decrease mortality and childhood disability, and is now the standard of care [1]. About $23-29 \%$ of infants with

The study was presented as an oral presentation at 34th Annual Children's National Symposium: ECMO and the Advanced Therapies for Respiratory Failure.

Prashant Agarwal

pagarwal@dmc.org

1 Division of Neonatal-Perinatal Medicine, Department of Pediatrics, Children's Hospital of Michigan/Wayne State University, Detroit, MI, USA

2 Department of Radiology, Children's Hospital of Michigan, Detroit, MI, USA

3 Division of Neonatal-Perinatal Medicine, Department of Pediatrics, University of Mississippi Medical Center, Jackson, MS, USA

4 Department of General Surgery, Children's Hospital of Michigan, Detroit, MI, USA
HIE develop persistent pulmonary hypertension (PPHN) [1-3]. The potential mechanisms for development of PPHN in infants with HIE include: in-utero and perinatal hypoxia and acidosis, ventricular dysfunction and lung pathology such as meconium aspiration syndrome and pulmonary hemorrhage [4-7]. Of the infants with HIE and PPHN, 49\% need extracorporeal membrane oxygenation (ECMO) therapy [1, 3]. However, these infants also often have multiorgan dysfunction and coagulopathy [8] and are at high risk of intracranial hemorrhage with anticoagulation during ECMO.

Data on survival and outcomes in infants with HIE who receive therapeutic hypothermia and ECMO are limited. The objective of our study was to evaluate in-hospital outcomes, including-intracranial hemorrhage, survival to discharge, and severity of brain injury on magnetic resonance imaging (MRI) in this patient population.

\section{Methods}

This was a single-center retrospective cohort study from January 2005 to December 2016 of infants admitted to a level IV Neonatal Intensive Care Unit (NICU). The study was approved and consent waived by the Institutional 
Review Board of Wayne State University. Inclusion criteria were: Gestational age $\geq 36$ weeks, therapeutic hypothermia for moderate to severe HIE, and treatment with ECMO for PPHN. Moderate or severe HIE was defined using the Eunice Kennedy Shriver National Institute of Child Health and Human Development (NICHD) screening biochemical criteria along with neurologic examination (modified Sarnat's staging) consistent with moderate or severe encephalopathy or seizures [1]. At our center, for infants with moderate or severe HIE, whole-body therapeutic hypothermia to $33.5^{\circ} \mathrm{C}$ for $72 \mathrm{~h}$ is initiated within $6 \mathrm{~h}$ of age via the Cincinnati Subzero Hypothermia system. Rewarming is done at $0.5{ }^{\circ} \mathrm{C}$ per hour until the esophageal temperature is $\geq 36.5{ }^{\circ} \mathrm{C}$ for $4 \mathrm{~h}$. The diagnosis of PPHN was based on lability in oxygenation, along with echocardiographic evidence of pulmonary hypertension (i.e., elevated right ventricular systolic pressure, flattened or bowed ventricular septum, or right-to-left or bidirectional shunt at the atrial or ductal level). Inhaled Nitric Oxide is initiated for hypoxemic respiratory failure with oxygenation index $>20$ and ECMO for inability to maintain normal oxygen saturations or oxygenation index $>40$ despite optimal ventilatory and medical management. Refractory hypotension is also considered an indication for ECMO. All infants receive a head ultrasonography (HUS) to exclude intracranial hemorrhage prior to placement on ECMO and daily HUS to monitor for development of intracranial hemorrhage while on ECMO. All MRI were reviewed by a single pediatric neuroradiologist (DA) blinded to the clinical outcome. MRI were classified as per the NICHD pattern for brain injury: score of 0 for normal MRI; 1A for minimal cerebral lesions only; 1B for more extensive cerebral lesions without basal ganglia and thalamus (BGT), or posterior limb of internal capsule (PLIC) or anterior limb of internal capsule (ALIC) involvement and no area of watershed infarction; $2 \mathrm{~A}$ for any BGT, PLIC, or ALIC involvement or watershed infarction without any cerebral lesions; 2B for any BGT, PLIC, or ALIC involvement or watershed infarction with additional cerebral lesions; and 3 for cerebral hemispheric devastation [9].

\section{Results}

Our cohort $(n=20)$ received therapeutic hypothermia for moderate $(n=12)$, severe $(n=5)$, or undocumented severity $(n=3)$ of HIE. Meconium aspiration syndrome was the most common cause of PPHN $(n=10,50 \%)$. All infants received inhaled nitric oxide before progression to ECMO. Fifteen neonates had pre-ECMO oxygenation index $>40$, three neonates had oxygenation index in 30 s with refractory hypotension and two neonates received ECMO after completion of therapeutic hypothermia due to inability to maintain normal oxygen saturations and severe hypotension. Majority of infants received multiple blood products before ECMO. Mean (SD) age at initiation of ECMO and duration of ECMO were 4.4 (2.6) days and 7.3 (3.4) days, respectively. Eleven (58\%) infants underwent ECMO while receiving therapeutic hypothermia.

During ECMO, 30\% $(n=6)$ infants developed intracranial hemorrhage at a median (IQR) duration of 24 (20) hours following anticoagulation. The intracranial bleed was most commonly parenchymal hemorrhage $(n=4)$, with 1 case each of intraventricular and subdural hemorrhage. ECMO was discontinued on five of these six infants; two of whom survived to discharge.

Veno-arterial (VA) ECMO was performed in $12(60 \%)$ infants and the remaining $(n=8,40 \%)$ underwent venovenous(VV) ECMO. Five of six infants who developed intracranial hemorrhage underwent VA ECMO and all four infants who died underwent VA ECMO. Sixteen $(80 \%)$ infants survived to discharge with a mean (SD) length of hospitalization of 45.6 (14.6) days. Of the four infants who died, three had developed intracranial hemorrhage leading to discontinuation of ECMO and one had withdrawal of life-support after parental discussion due to high likelihood of poor neurologic outcome. Neonatal characteristics hospital course are described in Table 1.

Among the 16 surviving infants, 15 (94\%) had MRI; 7 (44\%) were normal, 3 (20\%) had intracranial hemorrhage, and $2(13 \%)$ each had NICHD score 1 and 2, while $1(7 \%)$ infant had NICHD score 3 pattern of brain injury, respectively. Of the seven infants with normal MRI, six had received VV ECMO. MRI findings are described in Table 2. Among survivors, $87.5 \%(n=14)$ were discharged on room air. Figure 1 describes short-term outcomes by severity of HIE and type of ECMO.

\section{Discussion}

About a third of infants with HIE develop PPHN, of whom 4-9\% need ECMO therapy. These infants are at high risk of intracranial hemorrhage during ECMO due to multiorgan dysfunction and coagulopathy. In this study, we evaluated in-hospital outcomes of 20 infants with HIE who received therapeutic hypothermia and ECMO. We found that 30\% infants developed intracranial hemorrhage during ECMO and $80 \%$ survived to discharge. Among survivors, $44 \%$ had normal MRI.

In a survey published in 2009, of all, $40 \%$ responders from active neonatal ECMO centers that were part of ELSO registry said they would never or rarely offer ECMO to a patient with moderate HIE, and $48 \%$ would never offer ECMO to a neonate with severe HIE [10]. However, the number of neonates with HIE receiving ECMO has 
Table 1 Neonatal characteristics and hospital course

\begin{tabular}{|c|c|}
\hline \multicolumn{2}{|l|}{ Characteristic } \\
\hline Gestational age — weeks, median (IQR) & $38.5(37-40.25)$ \\
\hline \multicolumn{2}{|l|}{ Race - no. } \\
\hline Black & $13(65 \%)$ \\
\hline White & $3(15 \%)$ \\
\hline Other & $4(20 \%)$ \\
\hline Male gender $-n$ & $12(60 \%)$ \\
\hline Birth weight $-\mathrm{g}$, median (IQR) & $3385(2664-4023)$ \\
\hline Intubation in delivery room - no. & $17(85 \%)$ \\
\hline $\mathrm{CPR}$ in delivery room - no. & $10(50 \%)$ \\
\hline 5-minute Apgar score $<5-n$ & $15(75 \%)$ \\
\hline \multicolumn{2}{|l|}{ Cord blood, median (IQR) } \\
\hline $\mathrm{pH}$ & $6.87(6.75-6.98)$ \\
\hline Base deficit $-\mathrm{mmol} / \mathrm{liter}$ & $17.7(15.5-21.5)$ \\
\hline Clinical seizures - no. & $9(45 \%)$ \\
\hline \multicolumn{2}{|l|}{ Severity of encephalopathy - no. } \\
\hline Severe encephalopathy & $5(25 \%)$ \\
\hline Moderate encephalopathy & $12(60 \%)$ \\
\hline Unknown & $3(15 \%)$ \\
\hline \multicolumn{2}{|l|}{ Co-morbidities - no. } \\
\hline Meconium aspiration syndrome & $10(50 \%)$ \\
\hline Pulmonary hemorrhage & $3(15 \%)$ \\
\hline Culture proven sepsis & $2(10 \%)$ \\
\hline $\begin{array}{l}\text { Age at starting Inhaled Nitric } \\
\text { Oxide }-\mathrm{hr} \text {, median (IQR) }\end{array}$ & $9(5-67.5)$ \\
\hline Inotropic support — no. & $20(100 \%)$ \\
\hline Thrombocytopenia before ECMO- no. & $14(70 \%)$ \\
\hline \multicolumn{2}{|l|}{ Transfusion of blood products before ECMO } \\
\hline Packed red blood cells - no. & $13(65 \%)$ \\
\hline Platelets — no. & $14(70 \%)$ \\
\hline Fresh frozen plasma - no. & $18(90 \%)$ \\
\hline Cryoprecipitate - no. & $11(55 \%)$ \\
\hline \multicolumn{2}{|l|}{ Extraporporeal membrance oxygenation } \\
\hline Age at initiation $-\mathrm{hr}$, median (IQR) & $69(31-93.5)$ \\
\hline Initiated during cooling - no. & $11(55 \%)$ \\
\hline VA ECMO — no. & $12(60 \%)$ \\
\hline Intracranial hemorrhage - no. & $6(30 \%)$ \\
\hline Total duration — days, median (IQR) & $7(6-10)$ \\
\hline Days on mechanical ventilation, median (IQR) & $21.5(13.3-28.3)$ \\
\hline Length of stay — days, median (IQR) & $38.5(28-53.5)$ \\
\hline Death — no. & $4(20 \%)$ \\
\hline Age at death - days, median (IQR) & $6.5(3.8-7)$ \\
\hline MRI performed — no. & $15(75 \%)$ \\
\hline Age at MRI — days, median (IQR) & $29(17-40)$ \\
\hline \multicolumn{2}{|l|}{ Discharge status - no. } \\
\hline Gavage feeding & $12(60 \%)$ \\
\hline Anticonvulsant at discharge & $2(10 \%)$ \\
\hline Oxygen at discharge & $2(10 \%)$ \\
\hline Ventilator at discharge & 0 \\
\hline Ventriculo-Peritoneal shunt & $1(5 \%)$ \\
\hline
\end{tabular}

increased significantly since then [11], highlighting the importance of studying outcomes in this high-risk population.

Data on outcomes of infants with HIE receiving ECMO is limited. Massaro et al. described a case series of five
Table 2 Magnetic resonance imaging (MRI) findings

\begin{tabular}{lc}
\hline MRI results $(n=15)$ & \\
\hline MRI NICHD Score - no. & $7(46.7 \%)$ \\
0 & $1(6.7 \%)$ \\
$1 \mathrm{~A}$ & $1(6.7 \%)$ \\
$1 \mathrm{~B}$ & 0 \\
$2 \mathrm{~A}$ & $2(13.3 \%)$ \\
2B & $1(6.7 \%)$ \\
3 & $3(20 \%)$ \\
Intracranial hemorrhage &
\end{tabular}

infants with HIE receiving ECMO during therapeutic hypothermia [12]. Three out of these five infants developed intracranial hemorrhage, all survived to discharge and three were developmentally age appropriate at follow up. Antecedents and characteristics of infants with HIE enrolled in the Induced hypothermia and the standard cooling arm of the Optimizing Cooling strategies randomized controlled trials of the NICHD Neonatal Research Network, who developed PPHN were reported in one study [7]. Among the 303 infants with moderate or severe HIE, 67 (22\%) developed PPHN, of whom 49 (73\%) survived to discharge. Among the 67 infants with PPHN, 8 received ECMO, and 7 of these infants survived to discharge. Guaman et al. reported bleeding complications and mortality among neonates with HIE who underwent ECMO, from Extracorporeal Life Support Organization (ELSO) registry 2005 to 2013 [11]. In this study, among the 78 neonates who received therapeutic hypothermia for HIE and ECMO, 17 (22\%) neonates developed neurologic complications (defined by ELSO as: clinically determined brain death, ultrasonographic or computed tomographic evidence of brain hemorrhage, or infarction) and $12(15 \%)$ died before hospital discharge. MRI data were not reported in this study. The $20 \%$ mortality in our cohort is comparable to the $12-15 \%$ rates in these two studies $[7,11]$ as well as the larger cohort of infants with HIE treated with therapeutic hypothermia [1, 13].

Our results indicate a possibility of decreased risk of intracranial hemorrhage and improved survival to discharge with VV ECMO compared to VA ECMO. Some studies in pediatric ECMO population have demonstrated increased risk of mortality and intracranial hemorrhage with VA ECMO compared to VV ECMO [14-16]. The loss of pulsatile arterial flow during VA ECMO could disturb cerebral autoregulation and contribute to increased risk of intracranial hemorrhage [17]. The most common indication for ECMO in infants with HIE is respiratory pathology [11] and thus initiation with VV ECMO should be feasible in the majority of patients. However, there is further need to 


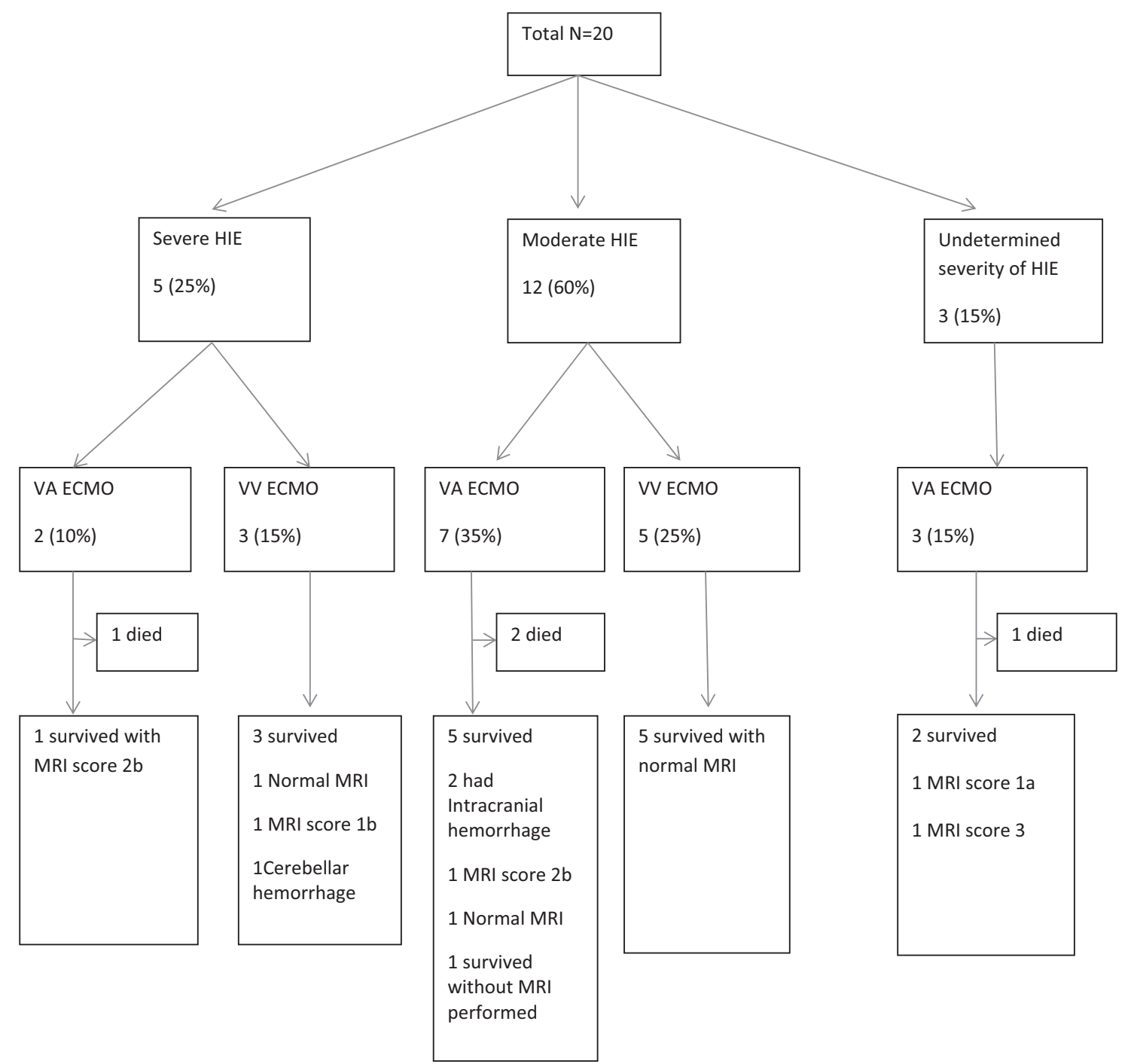

Fig. 1 Flow diagram depicting in-hospital outcomes by severity of HIE and type of ECMO

systematically study benefits of VV vs VA ECMO in infants with HIE.

There are several strengths to our study. All MRIs were read by a single pediatric neuroradiologist, blinded to the clinical data and scored as per the NICHD pattern of brain injury, which has been shown to correlate with death or disability at 2 years and also at 5-6 years [9, 18]. To our knowledge, this is the largest data set of neonates with HIE receiving therapeutic hypothermia and ECMO, with in-hospital and MRI outcomes. The major limitation is that the study is retrospective and from a single center. Three neonates with HIE from 2005 to 2006 did not have well documented neurological exam and hence severity of encephalopathy could not be determined for them. There is also possibility of selection bias, with some neonates with HIE not considered to be a candidate for ECMO due to high likelihood of poor neurologic outcome. Another limitation is the range of timing of MRI.
Finally, long-term neurologic outcome data were not available.

\section{Conclusion}

In the high-risk population of neonates with moderate or severe HIE, who required ECMO for PPHN, the $80 \%$ rate of survival to discharge home and $44 \%$ rate of normal MRI among survivors is encouraging. Long-term neurodevelopmental followup for HIE with PPHN requiring ECMO is needed.

Acknowledgements We express our gratitude to our medical and nursing colleagues, and to the infants and their families who participated in this study.

Author contributions All authors contributed to study concept and design, data acquisition and analysis, and drafting or critical revision of the manuscript. 


\section{Compliance with ethical standards}

Conflict of interest The authors declare that they have no conflict of interest.

Publisher's note: Springer Nature remains neutral with regard to jurisdictional claims in published maps and institutional affiliations.

\section{References}

1. Shankaran S, Laptook AR, Ehrenkranz RA, Tyson JE, McDonald SA, Donovan EF, et al. Whole-body hypothermia for neonates with hypoxic-ischemic encephalopathy. N Engl J Med. 2005;353:1574-84.

2. Shankaran S, Pappas A, Laptook AR, McDonald SA, Ehrenkranz RA, Tyson JE, et al. Outcomes of safety and effectiveness in a multicenter randomized, controlled trial of whole-body hypothermia for neonatal hypoxic-ischemic encephalopathy. Pediatrics. 2008;122:e791-8.

3. Shankaran S, Laptook AR, Pappas A, McDonald SA, Das A, Tyson JE, et al. Effect of depth and duration of cooling on deaths in the NICU among neonates with hypoxic ischemic encephalopathy: a randomized clinical trial. JAMA. 2014;312:2629-39.

4. Lapointe A, Barrington KJ. Pulmonary hypertension and the asphyxiated newborn. J Pediatr. 2011;158(2 Suppl):e19-24.

5. Lakshminrusimha S. The pulmonary circulation in neonatal respiratory failure. Clin Perinatol. 2012;39:655-83.

6. Aggarwal S, Natarajan G. Biventricular function on early echocardiograms in neonatal hypoxic-ischaemic encephalopathy. Acta Paediatr. 2017;106:1085-90.

7. Lakshminrusimha S, Shankaran S, Laptook A, McDonald S, Keszler M, Van Meurs K. et al. Pulmonary hypertension associated with hypoxic-ischemic encephalopathy-antecedent characteristics and comorbidities. J Pediatr. 2018;196:45-51.

8. Jacobs SE, Berg M, Hunt R, Tarnow-Mordi WO, Inder TE, Davis PG. Cooling for newborns with hypoxic ischaemic encephalopathy. Cochrane Database Syst Rev. 2013;31:CD003311.

9. Shankaran S, Barnes PD, Hintz SR, Laptook AR, Zaterka-Baxter $\mathrm{KM}$, McDonald SA, et al. Brain injury following trial of hypothermia for neonatal hypoxic-ischaemic encephalopathy. Arch Dis Child Fetal Neonatal Ed. 2012;97:F398-404.

10. Chapman RL, Peterec SM, Bizzarro MJ, Mercurio MR. Patient selection for neonatal extracorporeal membrane oxygenation: beyond severity of illness. J Perinatol. 2009;29:606-11.

11. Cuevas Guaman M, Lucke AM, Hagan JL, Kaiser JR. Bleeding complications and mortality in neonates receiving therapeutic hypothermia and extracorporeal membrane oxygenation. Am J Perinatol. 2018;35:271-6.

12. Massaro A, Rais-Bahrami K, Chang T, Glass P, Short BL, Baumgart S. Therapeutic hypothermia for neonatal encephalopathy and extracorporeal membrane oxygenation. J Pediatr. 2010;157:499-501, e1.

13. Shankaran S, Laptook AR, Pappas A, McDonald SA, Das A, Tyson JE, et al. Effect of depth and duration of cooling on death or disability at age 18 months among neonates with hypoxicischemic encephalopathy: a randomized clinical trial. JAMA. 2017;318:57-67.

14. Skinner SC, Iocono JA, Ballard HO, Turner MD, Ward AN, Davenport DL, et al. Improved survival in venovenous vs venoarterial extracorporeal membrane oxygenation for pediatric noncardiac sepsis patients: a study of the Extracorporeal Life Support Organization registry. J Pediatr Surg. 2012;47: 63-7.

15. Carpenter JL, Yu YR, Cass DL, Olutoye OO, Thomas JA, Burgman $\mathrm{C}$, et al. Use of venovenous ECMO for neonatal and pediatric ECMO: a decade of experience at a tertiary children's hospital. Pediatr Surg Int. 2018;34:263-8.

16. Cashen K, Reeder RW, Shanti C, Dalton HJ, Dean JM, Meert KL, et al. Is therapeutic hypothermia during neonatal extracorporeal membrane oxygenation associated with intracranial hemorrhage? Perfusion. 2018;33:354-62.

17. de Mol AC, Liem KD, van Heijst AF. Cerebral aspects of neonatal extracorporeal membrane oxygenation: a review. Neonatology. 2013;104:95-103.

18. Shankaran S, McDonald SA, Laptook AR, Hintz SR, Barnes PD, Das A, et al. Neonatal magnetic resonance imaging pattern of brain injury as a biomarker of childhood outcomes following a trial of hypothermia for neonatal hypoxic-ischemic encephalopathy. J Pediatr. 2015;167:987-93e3. 